\title{
A Differential Chaotic Bit-Interleaved Coded Modulation System Over Multipath Rayleigh Channels
}

Jia Zhan, Lin Wang, Senior Member, IEEE, Marcos Katz, Member, IEEE, and Guanrong Chen, Fellow, IEEE

\begin{abstract}
In this paper, a novel differential chaotic bitinterleaved coded modulation (DC-BICM) system is proposed for band-limited transmission. This system combines protographbased low density parity check codes with constellation-based $M$-ary differential chaos shift keying (DCSK) modulation by one bitwise interleaving. Bit error rate simulation results show that the system has higher coding gain compared with the constellation-based $M$-ary DCSK modulation system with the same spectral efficiency over multipath Rayleigh fading channels. At the same time, several simulations and P-EXIT analysis are used to analyze the performance of the proposed system. It is found that there is a lot of room for optimization of the system by comparing decoding thresholds and simulation results. Moreover, the system with only partial channel state information has better performance and lower complexity compared with the traditional bit-interleaved coded modulation (BICM) directsequence-spread-spectrum system. As a result, the DC-BICM system is a good candidate for band-limited transmission.
\end{abstract}

Index Terms-Constellation-based $M$-ary DCSK, BICM, protograph-based LDPC, multipath Rayleigh fading channel, band-limited transmission.

\section{INTRODUCTION}

C HAOTIC modulation has an excellent anti-interference ability with some unique properties, such as non-periodic signals, low correlation, noise-like, deterministic and wideband. Differential chaos shift keying (DCSK) [1], [2] is proposed to overcome the synchronization problem in chaotic communication systems, which can offer excellent performance over multipath fading channels [3], [4].

However, DCSK has low data rate and bandwidth efficiency because one half of the bit duration is spent on sending non-information-bearing reference samples. Several different methods have been proposed to solve this problem. $M$-ary DCSK [5], which employs orthogonal Walsh code sequences

This work was supported in part by the NSF of China under Grant 61271241 and Grant 61671395 and in part by the Hong Kong Research Grants Council under GRF under Grant CityU 11208515. The associate editor coordinating the review of this paper and approving it for publication was J. Choi.(Corresponding author: Lin Wang.)

J. Zhan and L. Wang are with the Department of Communication Engineering, Xiamen University, Fujian 361005, China (e-mail: wanglin@xmu.edu.cn).

M. Katz is with the Centre for Wireless Communications, University of Oulu, 90014 Oulu, Finland (e-mail: marcos.katz@ee.oulu.fi).

G. Chen is with the Department of Electronic Engineering, City University of Hong Kong, Hong Kong (e-mail: eegchen@cityu.edu.hk). to transmit multi-bits, provides an optimal solution over an AWGN channel with non-coherent detection of chaotic signals by employing the Generalized Maximum Likelihood (GML) decision rule [6], and its improved scheme named MCS-DCSK [7] has lower complexity and higher bandwidth efficiency. Recently, a new $M$-ary OM-DCSK modulation using both T-R technique and $M$-ary orthogonal modulation was proposed [8], which has outstanding performances. Differing from these methods, generalized constellation-based $M$-ary DCSK modulation proposed in [9], as an extension of QCSK [10], the circle-constellation-based M-ary DCSK system is appropriate for band-limited communication systems similarly to the $M$-ary PSK-DCSK [11], providing better performances than PSK-DCSK. However, its performance decreases with the increasing level $M$ [12]. The square-constellation-based (s-c-b) $M$-ary DCSK communication system has higher bandwidth-efficiency and peak-to-average-power ratio (PAPR) performance than the circle-constellation-based $M$-DCSK system [9].

As is well known, the reliability and low power consumption are also very important measures for the performance of wireless communication systems, and the error correction coding can effectively improve reliability and reduce power consumption [13]. It has been proved in [14]-[17] that error control codes can significantly improve the overall performance of chaos-based digital communication systems. However, channel coding can increase system bandwidth by producing redundancy, which is not good for band-limited transmission.

In order to achieve a good trade-off between spectral efficiency and power consumption for transmission systems, coded modulation is the best choice, which can achieve high coding gains without requiring extra bandwidths. BICM, firstly proposed in [19] and later analyzed in [20], is most popular because of its simplicity, good energy efficiency and spectrum efficiency over fading channels [21]. BICM can obtain maximum code-word dispersion degree by combining binary error-control codes and higher-order modulation with a bit-wise interleaving in an appropriate soft-decision bit metric. P-LDPC codes were applied to the BICM system over the Gaussian channel with a mapping scheme called variable degree matched mapping (VDMM) in [22], for which some optimized designs of the scheme were carried out in [23] and [24]. But, very few studies have been performed over multipath fading channels. 


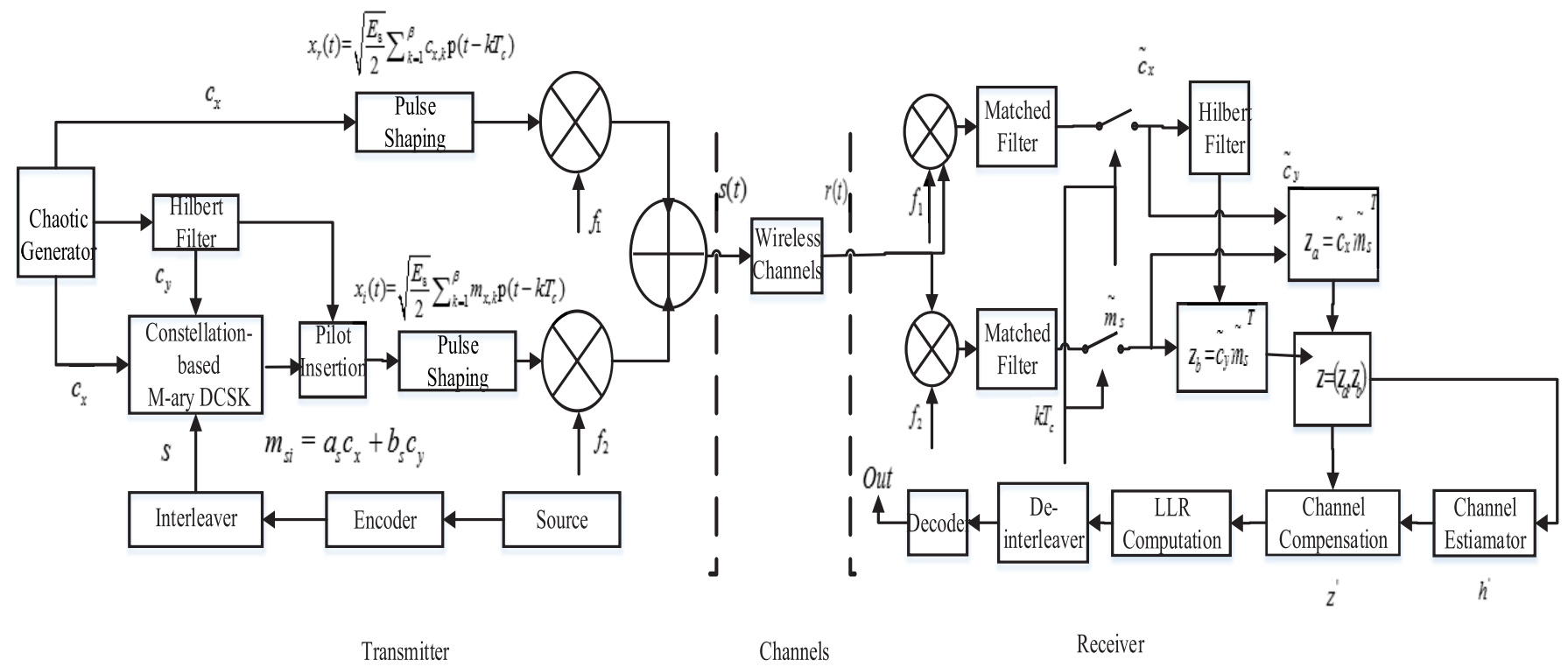

Fig. 1. The block diagram of the DC-BICM scheme.

Therefore, a differential chaotic bit-interleaved coded modulation system is proposed in this paper, aiming at improving coding gain without requiring extra bandwidths over multipath Rayleigh fading channels for band-limited transmission. P-LDPC is chosen as the error correction code of this system for its outstanding advantages [18], [29], which has to obtain the channel state information (CSI) because of using belief propagation (BP) iterative decoding algorithm [30]. But this system only needs to know partial CSI by using constellationbased $M$-ary DCSK modulation, which adopts non-coherent reception. Its design of two-dimensional constellation complies requires for joint coded modulation technique compared with other $M$-ary DCSK systems. The squared-constellationbased $M$-ary is selected as an example to verify the feasibility of the proposed system. The proposed system offers good antimultipath fading performance and high spectrum efficiency, and has lower complexity and better robustness compared with the traditional BICM-DS/SS system [25], [31].Therefore, it is suitable for band-limited channel scenarios with strong antiinterference ability when it is hard to obtain perfect CSI.

The contributions of this work can be summarized as follows:

1) The DC-BICM system is designed. The bit error rate (BER) curves demonstrate that the proposed system has higher coding gains compared with the squaredconstellation-based $M$-ary DCSK system with the same spectral efficiency over three-path fading channel, from which it can be concluded that the system has high spectral efficiency and strong ability to resist multipath interference.

2) There exist best spreading factor and best decoding iterations for the system performance according to the simulation results, and the performance gets better with the increasing code length, but, getting worse with increasing M. Also, the gap between simulation results and decoding thresholds signifies that there is a large room for optimization of the system.

3) The comparison between the proposed system and the traditional BICM-DS/SS system over multipath fading channels shows that the proposed system has better performance with partial CSI, which indicates that the proposed system has stronger robustness.

The remainder of this paper is organized as follows. Section II describes the system model and related notions. LLR calculation and P-EXIT analysis are presented in Section III. The simulation results and analysis are given in Section IV. Finally, Section V concludes the investigation.

\section{System Model and Related Notions}

In this section, the proposed DC-BICM communication system shown in Fig.1 is described in details. Here, square constellation is adopted as instance.

\section{A. Transmitter}

1) Squared-Constellation-Based M-ary DCSK: The squared-constellation-based $M$-ary DCSK is based on the principle of square constellation, where the 4-ary DCSK modulation is same as the QCSK modulation, and the constellation of the squared-constellation-based $M$-ary DCSK is shown in Fig.2.

2) Encoder: Accumulate repeat jagged accumulate (ARJA) codes [18] are applied to the proposed system. There are two ARJA code families constructed by different ways as shown in Fig.3(a) and Fig.3(b), respectively. Here, $n$ denotes the number of variable nodes, and the number of the not-punched variable nodes is $\tilde{n}$, which must equal to $\log _{2} M$ according to the variable degree matched mapping (VDMM) rule [22]. Therefore, appropriate code type and code rate both must be selected according to $M$.

Assumed that $M$ is equal to 16 , ARJA with $1 / 2$ rate shown in Fig.3(a) is selected for coding with the corresponding mapping 


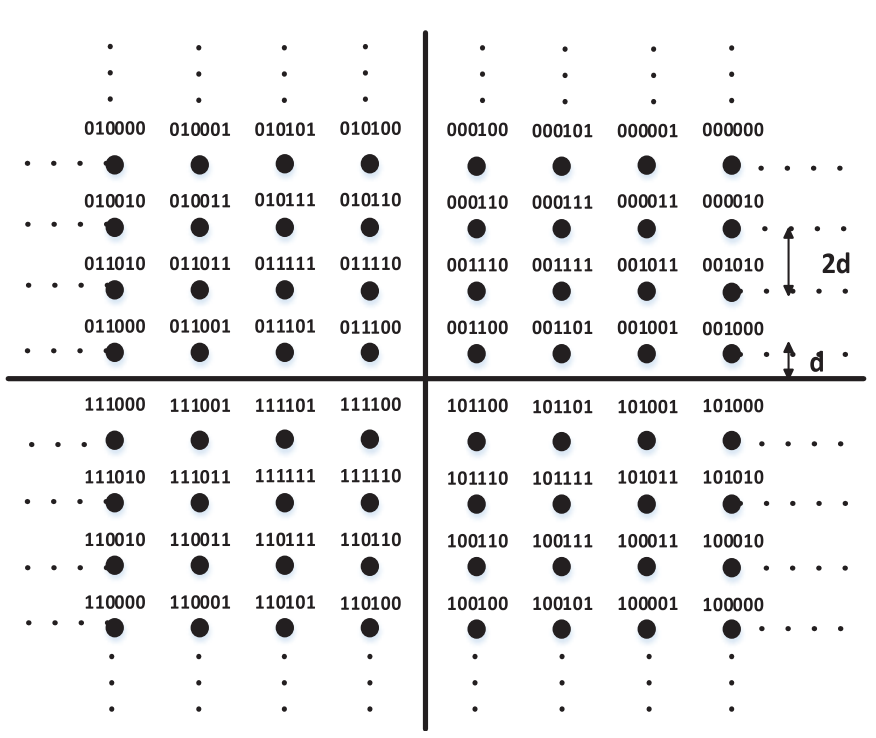

Fig. 2. The constellation of squared-constellation-based $M$-ary DCSK.

rules, namely, the number of not-punched variable nodes is just 4 . Bit-wise mutual information (MI) $I\left(x^{e_{i}} ; y\right)$ [26] in the constellation needs to be computed. Doing this for the case of gray-labeled squared-constellation-based 16-ary DCSK reveals $I\left(x^{e_{0}} ; y\right)=I\left(x^{e_{1}} ; y\right)$ and $I\left(x^{e_{2}} ; y\right)=I\left(x^{e_{3}} ; y\right)$ with $I\left(x^{e_{1}} ; y\right)>I\left(x^{e_{3}} ; y\right)$. The term water-filling refers to the mapping of variable node degrees in proportion to the bit-plane MI, which still performs well because higher-degree nodes can affect more other nodes than lower-degree nodes although it is not optimal [23].

Firstly, encoder generates code bits. Then, the code bits $\left(c_{1}, c_{2}, c_{3}, c_{4}\right)$ corresponding to variable nodes $\left(V_{2}, V_{4}, V_{1}, V_{3}\right)$ in the ARJA protograph are mapped to the signal labeling $\left(x^{e_{0}}, x^{e_{1}}, x^{e_{2}}, x^{e_{3}}\right)$ in the s-c-b 16-ary DCSK constellation according to the water-filling, which is shown in Fig.4.

Secondly, the chaotic generator generates a chaotic reference signal $\boldsymbol{c}_{x}=c_{x, 1}, c_{x, 2}, \ldots, c_{x, \beta}$ from the map

$$
x_{k+1}=1-2 x_{k}^{2} .
$$

which has a length of $\beta$, where $\beta$ is defined as the spreading factor. For a normalized chaotic signal with zero mean, the variance is equal to one. Then, $\boldsymbol{c}_{\boldsymbol{x}}$ is transformed into another quadrature signal $\boldsymbol{c}_{\boldsymbol{y}}=c_{y, 1}, c_{y, 2}, \ldots c_{y, \beta}$ by using Hilbert transform, thus two independent orthogonal chaotic signals are generated. The information-bearing signal can be generated as a linear combination of the quadrature signals $\boldsymbol{c}_{\boldsymbol{x}}$ and $\boldsymbol{c}_{\boldsymbol{y}}$, in the form of $\boldsymbol{m}_{s}=a_{s} \boldsymbol{c}_{\boldsymbol{x}}+b_{s} \boldsymbol{c}_{\boldsymbol{y}}$, where the index $s$ identifies the symbol with a signal labeling $X$ in the signal space, $s=1,2, \ldots, M$, and $a_{s}$ and $b_{s}$ presents the real part and the image part corresponding to this symbols, respectively (see Fig.4 for the squared-constellation-based 16 -ary DCSK). During the $i$-th symbol duration, the output of the transmitter is

$$
s(t)=x_{r}(t) \cos \left(2 \pi f_{1} t+\phi_{1}\right)+x_{i}(t) \cos \left(2 \pi f_{2} t+\phi_{2}\right),
$$

$$
\begin{aligned}
& x_{r}(t)=\sqrt{\frac{E_{s}}{2}} \sum_{k=1}^{\beta} c_{x, k} p\left(t-k T_{c}\right), \\
& x_{i}(t)=\sqrt{\frac{E_{s}}{2}} \sum_{k=1}^{\beta} m_{x, k} p\left(t-k T_{c}\right),
\end{aligned}
$$

where $p(t)$ is the square-root-raised-cosine filter, $T_{c}$ is the chip time, $x_{r}(t)$ and $x_{i}(t)$ represent the chaotic reference signal and the information signals, respectively, and $\phi_{1}$ and $\phi_{2}$ denote the phase angles of the carrier modulation in which the two modulated carriers are orthogonal, $f_{1}$ and $f_{2}$ denote the frequencies of modulated carriers, and $E_{s}$ denotes the symbol energy equaling 1 . In the case of a square constellation with $M=4,16,64,256 \ldots$ and amplitudes of $\pm d, \pm 3 d, \pm 5 d, \ldots, \pm(\sqrt{M}-1) d$ on both directions, when considering the reference signal, the average symbol energy in the constellation is computed by

$$
E_{\text {avg }}=\frac{E_{s}}{2 M} \times \frac{2 M(M-1)}{3} d^{2}+\frac{E_{s}}{2} .
$$

\section{B. The Receiver}

The receiver signals is given by

$$
r(t)=\sum_{l=1}^{L} \alpha_{l} \delta\left(t-\tau_{l}\right) \otimes s(t)+\eta(t),
$$

where, $\otimes$ is the convolution operator, $\eta(t)$ is an AWGN with zero mean and power spectral density $N_{0} / 2, L$ is the number of paths, and $\alpha_{l}$ and $\tau_{l}$ are the channel coefficient and the path delay of the $l$-th path, respectively.

In this channel model, the Rayleigh probability density function (PDF) of the channel coefficient $\alpha_{l}$ is given by

$$
f(x)=\frac{\alpha_{l}}{\sigma^{2}} \exp \left(-\frac{\alpha_{l}^{2}}{2 \sigma^{2}}\right), \alpha_{l}>0 .
$$

where $\sigma>0$ is the scale parameter of the distribution. A three-path Rayleigh fading channel with $E\left[\alpha_{0}^{2}\right]=\frac{1}{2}$, $E\left[\alpha_{1}^{2}\right]=\frac{2}{5}, E\left[\alpha_{2}^{2}\right]=\frac{1}{10}, \tau_{0}=0, \tau_{1}=2, \tau_{3}=5$ is applied.

The received signal $r(t)$ is first separated by two corresponding modulated carrier frequencies. The separated signals are filtered by two corresponding matched filters. Then, the two filtered signals are sampled every $k T_{c}$ time unit. This way, the desired signals are demodulated, $\tilde{\boldsymbol{c}}_{\boldsymbol{x}}$ and $\tilde{\boldsymbol{m}}_{\boldsymbol{s}}$ denote chaotic reference signal and informational signal, respectively. Then, $\tilde{\boldsymbol{c}}_{\boldsymbol{x}}$ is transformed into $\tilde{\boldsymbol{c}}_{\boldsymbol{y}}$ by the Hilbert transform. Here, the received symbol $z=(z a, z b)$, with

$$
\begin{aligned}
z a \approx & \sum_{l=1}^{L} \alpha_{l}^{2} a_{s} E_{s} / 2+\sum_{k=1}^{\beta} \sum_{l=1}^{L} \alpha_{l} \sqrt{E_{S} / 2} c_{x, k-\tau_{l}} \eta_{k+\beta} \\
& +\sum_{k=1}^{\beta} \sum_{l=1}^{L} \alpha_{l} \sqrt{E_{S} / 2}\left(a_{s} c_{x, k-\tau_{l}}+b_{s} c_{y, k-\tau_{l}}\right) \eta_{k} \\
& +\sum_{k=1}^{\beta} \eta_{k} \eta_{\beta+k},
\end{aligned}
$$




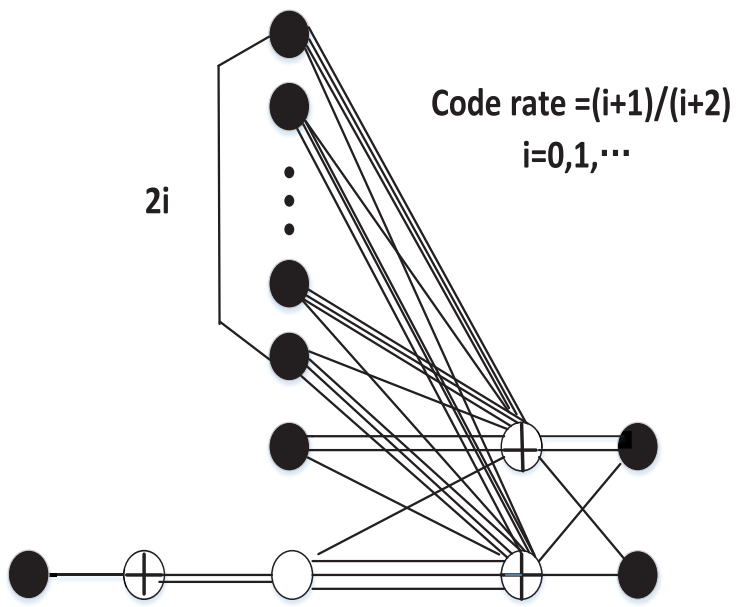

(a) Protograph of ARJA family with rates $1 / 2$ and higher.

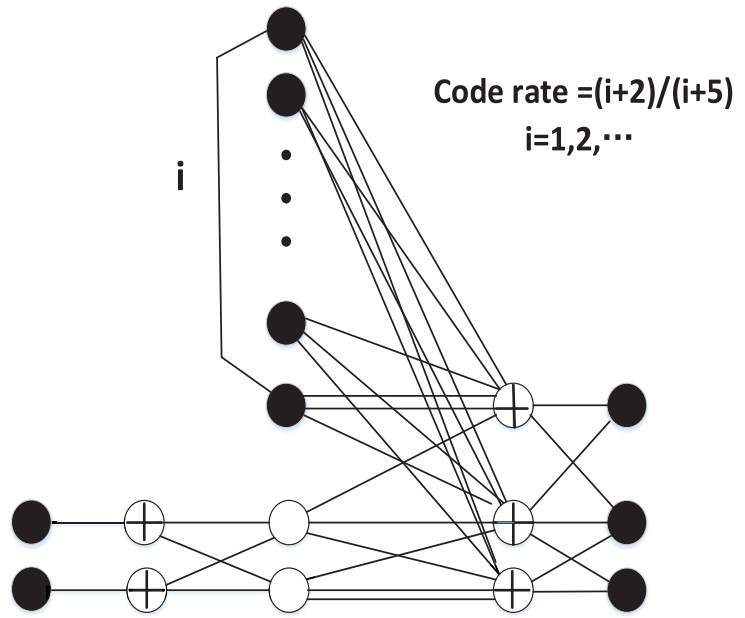

(b) Protograph of ARJA-5-checks family with rates $1 / 2$ and higher.

Fig. 3. ARJA codes.

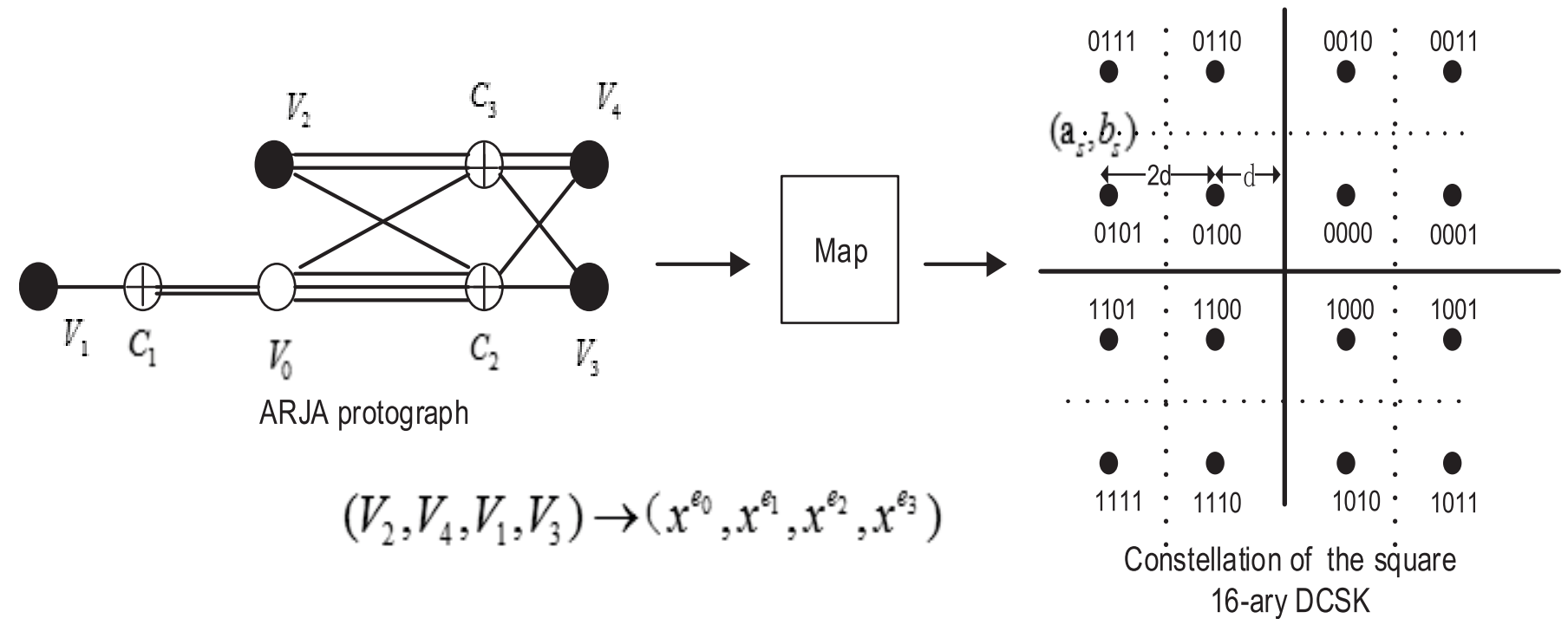

Fig. 4. An example of the VDMM mapper approach for the DC-BICM system with ARJA rate 0.5 and $M$ equal to 16 .

$$
\begin{aligned}
z b \approx & \sum_{l=1}^{L} \alpha_{l}^{2} b_{s} E_{s} / 2+\sum_{k=1}^{\beta} \sum_{l=1}^{L} \alpha_{l} \sqrt{E_{s} / 2} c_{x, k-\tau_{l}} \eta_{k+\beta} \\
& +\sum_{k=1}^{\beta} \sum_{l=1}^{L} \alpha_{l} \sqrt{E_{s} / 2}\left(a_{s} c_{x, k-\tau_{l}}+b_{s} c_{y, k-\tau_{l}}\right) \tilde{\eta}_{k} \\
& +\sum_{k=1}^{\beta} \tilde{\eta}_{k} \eta_{\beta+k} .
\end{aligned}
$$

\section{LLR COMPUTATION AND P-EXIT ANALYSIS}

In this section, LLR computation is carried out, and a P-EXIT analysis is introduced to evaluate the decoding thresholds and to analyze the system.

\section{A. LLR Computation}

Assume that the largest multipath delay is much shorter than the symbol duration, i.e., $0 \leq \tau_{l_{m} a x} \leq \beta$, hence the inter-symbol interference (ISI) is negligible. It was found [9] that the decision variables $z_{a}$ and $z_{b}$ are the Gauss variables with the means $m_{1}=\sum_{l=1}^{L} \alpha_{l}^{2} a_{s} \frac{E_{s}}{2}$ and $m_{2}=$ $\sum_{l=1}^{L} \alpha_{l}^{2} b_{s} \frac{E_{s}}{2}$, respectively, and the same variance $\sigma^{2}=$ $\sum_{l=1}^{L} \alpha_{l}^{2} \frac{E\left(a_{s}^{2}+b_{s}^{2}+1\right) E_{s} N_{0}}{4}$. Here, $N_{0}$ is the standard deviation of Gaussian noise. Defining $E\left(a_{s}^{2}+b_{s}^{2}+1\right) \frac{E_{s}}{2} \approx E_{a v g}$. Over the multipath Rayleigh fading channel, the decision variables $z^{\prime}=z /\left(h \cdot \frac{E_{s}}{2}\right), h=\sum_{l=1}^{L} \alpha_{l}^{2}$ denote the partial channel state information. If $\mathrm{h}$ is estimated, $h=h^{\prime}$. Then, the means $\overline{m_{1}}=a_{s}, \overline{m_{2}}=b_{s}$ and, the variance $\overline{\sigma^{2}}=$ $\left(h \times \frac{E_{a} v g N_{0}}{2}+\frac{\beta N_{0}^{2}}{4}\right) /\left(h \cdot \frac{E_{s}}{2}\right)^{2}$. Therefore, for the given symbol $\left(a_{s}, b_{s}\right)$, the probability density function of the observation signal $z^{\prime}=\left(z_{a}^{\prime}, z_{b}^{\prime}\right)$ is given by

$$
p\left(z^{\prime} \mid s\right)=\frac{1}{2 \pi \bar{\sigma}^{2}} \exp \left(-\frac{\left(z_{a}^{\prime}-\overline{m_{1}}\right)^{2}+\left(z_{b}^{\prime}-\overline{m_{2}}\right)^{2}}{2 \bar{\sigma}^{2}}\right) .
$$




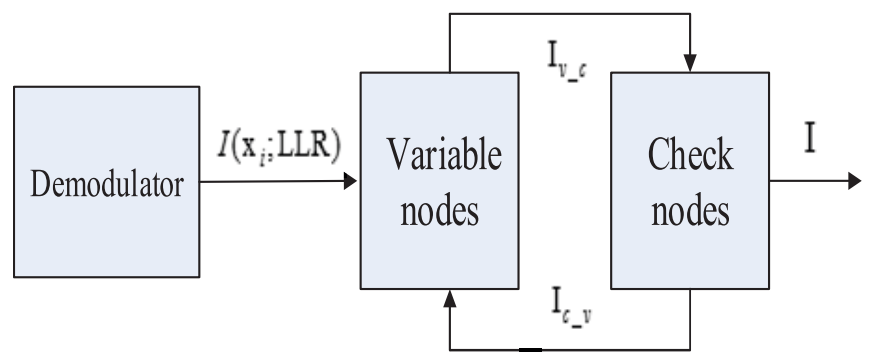

Fig. 5. Block diagram of the P-EXIT algorithm.

Here, Gaussian approximation $(G A)$ method is used to compute the LLR for the $i$-th transmitted bit, via

$$
L L R[i]=\log \frac{\sum_{m: c_{i}(m)=0} p\left(z^{\prime} \mid s_{m}\right) p\left(s_{m}\right)}{\sum_{\bar{m}: c_{i}(\bar{m})=1} p\left(z^{\prime} \mid s_{\bar{m}}\right) p\left(s_{\bar{m}}\right)},
$$

where $c_{i}(m)$ returns the $i$-th bit associated with the $m$-th modulated symbol $s_{m}$ according to the VDMM mapping rule, $m=0,1, \ldots M-1$, and $p\left(s_{m}\right)$ is a prior probability of symbol $s_{m}$ [15], [16], but, in this paper, $p\left(s_{m}\right) / p\left(s_{\bar{m}}\right)=1$.

\section{B. Channel Estimation}

In this paper, the iterative decoding algorithm is adopted. Therefore, it is needed to acquire channel information. According to [9], LS estimator is applied. The pilot symbol $(1,0)$ is inserted into the information symbols every $Q$ symbol intervals. During the transmission time of $\mathrm{Q}$ symbols, the channel parameter is estimated as $h^{\prime}=z_{p} /\left(E_{S} / 2\right)$, where $z_{p}$ denotes the real component of the received symbol transmitted from the pilot $(1,0)$.

\section{P-EXIT Algorinthm}

The block diagram of P-EXIT algorithm is shown in Fig.5.

Firstly, the extrinsic mutual information (MI) of the demodulator is calculated for a fixed $E_{b} / N_{0}$ [23], [27]. Initialize the priori MI $I_{v}^{i j}=0$ and $I_{c}^{i j}=0$ for variable node $V_{i}$ and check node $C_{j}$, respectively, $i=0,1,2, \ldots n, j=0,1, \ldots m$, where $m$ and $n$ denote the number of check nodes and variable nodes in the protograph, respectively. For the $i$-th code bit $c_{i}$, the extrinsic MI is given by

$$
\begin{aligned}
I\left(c_{i} ; L L R\right)= & \frac{1}{2} \times \sum_{g=0,1} \int_{-\infty}^{+\infty} p_{L L R}(\xi \mid c i=g) \\
& \times \log _{2} \frac{2 p_{L L R}(\xi \mid c i=g)}{p_{L L R}\left(\xi \mid c i=0+p_{L L R}(\xi \mid c i=1)\right.} d_{\xi} .
\end{aligned}
$$

The conditional probability density functions (PDF) of extrinsic LLRs values $p_{L L R}(\xi \mid c i=g)$ is estimated by using Monte Carlo simulations [28], where $c_{i}$ is code bit, $g \in 0,1$. An ARJA code of information length 4096 and rate $1 / 2$ is applied to the system over the multipath Rayleigh fading channel, where $M=16, \beta=32$, and $E_{b} / N_{0}$ is $8.5 \mathrm{~dB}$ ( $E_{b}$ is the average energy per information bit).

Then, it is needed to collect the LLR values generated by simulations within each frame under the condition of

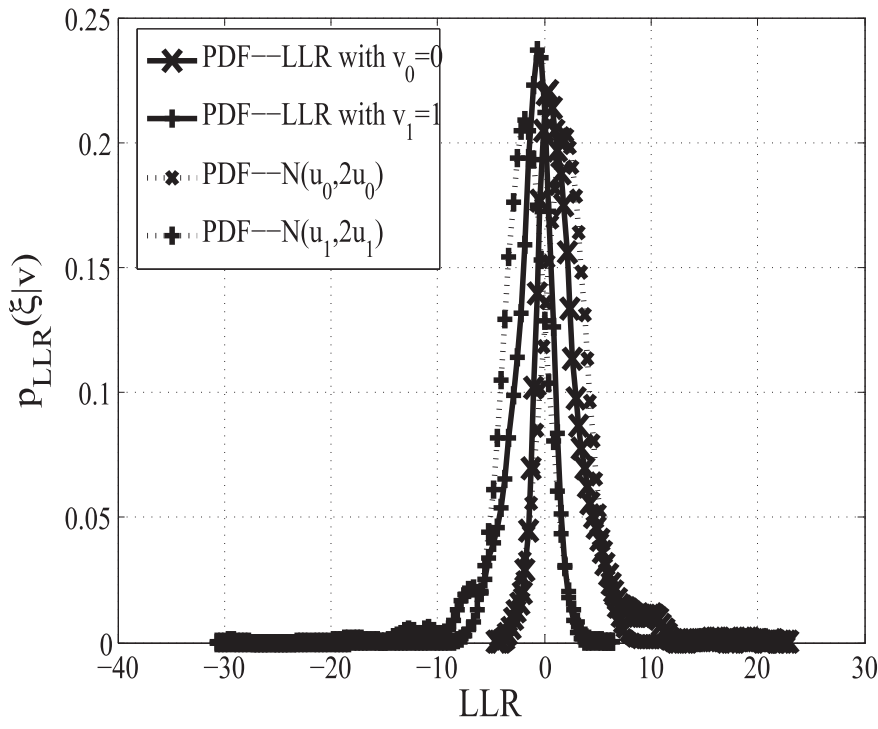

Fig. 6. Distribution of conditional PDFs of the output LLR values from demodulator with $\beta=32$ when the code bit $c_{i}$ corresponding to $V_{2}$ is equal to 0 and 1 , respectively.

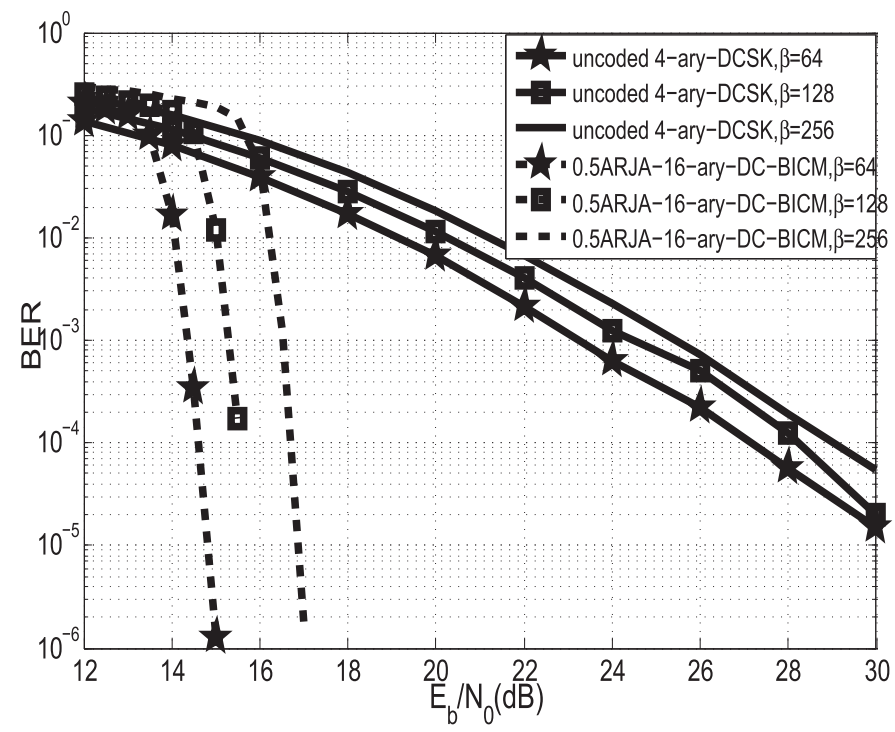

Fig. 7. Performance comparison between rate-1/2 ARJA 16-ary DC-BICM and squared-constellation-based 4-ary DCSK systems with same spectrum efficiency 2 bit/symbol.

$c_{i}=0,1, i=0,1, \ldots$ len $/ 5, c_{i}$ is the code bit corresponding to $V_{2}$, len is the code length, and plot the conditional probability density functions $(\mathrm{PDFs}) p_{L L R}\left(\xi \mid c_{i}=0\right)$ and $p_{L L R}\left(\xi \mid c_{i}=1\right)$. From Fig.6, it can be seen that $p_{L L R}\left(\xi \mid c_{i}=0\right)$ and $p_{L L R}\left(\xi \mid c_{i}=1\right)$ curves approximatively obey Gaussian distribution with $N\left(u_{0}, 2 u_{0}\right)$ and $N\left(u_{1}, 2 u_{1}\right)$, respectively. $u_{0}=E\left(L L R \mid c_{i}=0\right)$ and $u_{1}=E\left(L L R \mid c_{i}=1\right)$.

Simulations have also been performed for $c_{i}$ corresponding to different variable nodes $V_{i}, i=1,2,3,4$, and same results are obtained. Moreover, it was found by performing simulations on demodulator that the LLR values within each coded frame follow Gaussian distributions. However, the PDFs do not obey Gaussian distribution according to simulations when $\beta$ is not enough large, because the inter-symbol interference (ISI) cannot be ignored at this time. It can be obtained 


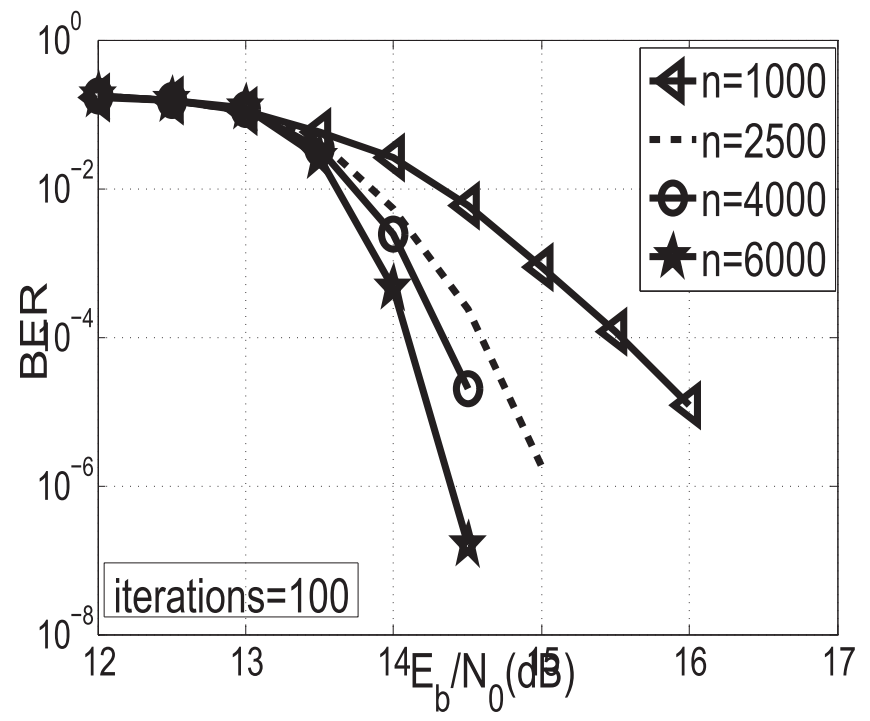

(a) different code lengths.

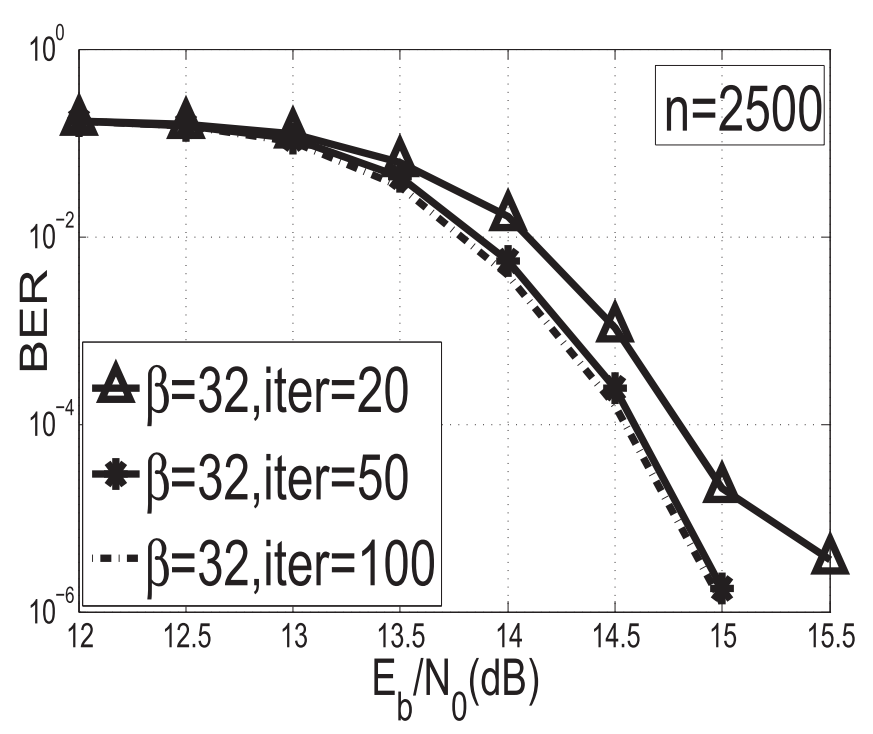

(b) different decoding iterations.

Fig. 8. Influences of the main parameters on the performance of the DC-BICM system.

from [16] and [28] that $p_{L L R}\left(\xi \mid V_{i}\right)$ follows Gaussian distribution when $\beta$ is very large, with

$$
\begin{aligned}
J\left(\sigma_{i}\right)= & I\left(V_{i} ; L L R\right)=1-\int_{-\infty}^{+\infty} \frac{\exp \left(-\frac{\left(\xi-\sigma_{i}^{2}\right)^{2}}{2 \sigma_{i}^{2}}\right)}{\sqrt{2 \pi \sigma_{i}^{2}}} \\
& \times \log _{2}[1+\exp (-\xi)] d_{\xi},
\end{aligned}
$$

where $\sigma_{i}^{2}=2 u_{i}, u_{i}$ is the average value of LLRs in the case of $\mathbf{c}=0$ or $\mathbf{c}=1$, and the code bit $\mathbf{c}$ is corresponding to $V_{i}$.

Secondly, set the MI at the input of variable nodes in line with the permutation pattern of VDMM, that is, $I_{c h}^{\pi(i)}=$ $I\left(V_{i} ; L L R\right), i=0,1, \ldots, n$. For $V_{0}$ that is punctured, $I_{c h}^{0}$ is set to 0 .

Thirdly, update the external mutual information from variable nodes to check nodes achieved by

$$
\begin{aligned}
I_{v c}^{i j}= & J\left(\sqrt{\sum_{t \neq j} b^{i, t}\left[J^{-1}\left(I_{v}^{i, t}\right)\right]^{2}+\left[J^{-1}\left(I_{c h}^{i}\right)\right]^{2}}\right), \\
& \left.+\left(b^{i, j}-1\right)\left[J^{-1}\left(I_{v}^{i, j}\right)\right]^{2}\right),
\end{aligned}
$$

where $i=0,1, \ldots, n, j=0,1, \ldots m$. If $b^{i j}=0$, then $I_{v c}^{i j}=0$, where $b^{i j}$ is the number of edges connecting the check node $C_{j}$ to the variable node $V_{i}$ in the protograph. Then, $I_{v c}^{i j}$ is assigned to prior MI of check nodes, namely $I_{c}^{i j}=I_{v c}^{i j}$.

To this end, update the external mutual information $I_{c v}$ from check nodes to variable nodes given by

$$
\begin{aligned}
I_{c v}^{i j}= & 1-J\left(\sqrt{\sum_{t \neq i} b^{t, j}\left[J^{-1}\left(1-I_{c}^{t, j}\right)\right]^{2}}\right. \\
& \left.+\left(b^{i, j}-1\right)\left[J^{-1}\left(1-I_{c}^{i, j}\right)\right]^{2}\right),
\end{aligned}
$$

where $i=0,1, \ldots, n, j=0,1, \ldots m$. If $b^{i j}=0$, then $I_{c v}^{i j}=0$. Set $I_{v}^{i j}=I_{c v}^{i j}$.
Finally, evaluate the posteriori probability log-likelihood ratio (APP-LLR) MI according to

$$
\begin{aligned}
I_{i} & =J\left(\sqrt{\sum_{t} b^{i, t}\left[J^{-1}\left(I_{v}^{i, t}\right)\right]^{2}+\left[J^{-1}\left(I_{c h}^{i}\right)\right]^{2}}\right), \\
i & =0,1, \ldots n .
\end{aligned}
$$

The maximum number of iterations is set to 100 , iterating from the second step until I equal to 1 , and the lowest $\left(E_{b} / N_{0}\right)^{*}$ satisfying this criterion is the lowest decoding thresholds.

\section{Simulation Results And Discussions}

To illustrate the effectiveness and advantages of the proposed DC-BICM system, extensive simulations have been conducted under a multipath Rayleigh fading channel with $E\left[\alpha_{0}^{2}\right]=\frac{1}{2}, E\left[\alpha_{1}^{2}\right]=\frac{2}{5}, E\left[\alpha_{2}^{2}\right]=\frac{1}{10}$ and $\tau_{0}=0, \tau_{1}=2$, $\tau_{3}=5$ as follows.

Firstly, Fig.7 compares the performances between the DC-BICM system (code length is 2500 and decoding iterations are 50) and the uncoded squared-constellation-based 4-ary DCSK system. A pair of curves indicate that DC-BICM system obtains almost $12.5 \mathrm{~dB}$ coding gain better than the uncoded squared-constellation-based 4-ary DCSK system with the same spectrum efficiency of $2 \mathrm{bit} / \mathrm{symbol}$ when BER and spreading factor $\beta$ are equal to $10^{-4}$ and 64 , respectively. It can be concluded from all of the above information that the system can not only get good performance for anti-multipath fading and also has high bandwidth efficiency.

Secondly, a series of BER curves of the DC-BICM system with different code lengths and 100 decoding iterations are displayed in Fig.8(a). Fig.8(b) presents a set of BER curves of the DC-BICM system with different decoding iterations and 2500 code lengths. It can be seen from Fig.8(a) that the performance of the system is better with increasing code length. It can be concluded that the code length has a great impact on the performance. The curves shown in Fig.8(b) also 


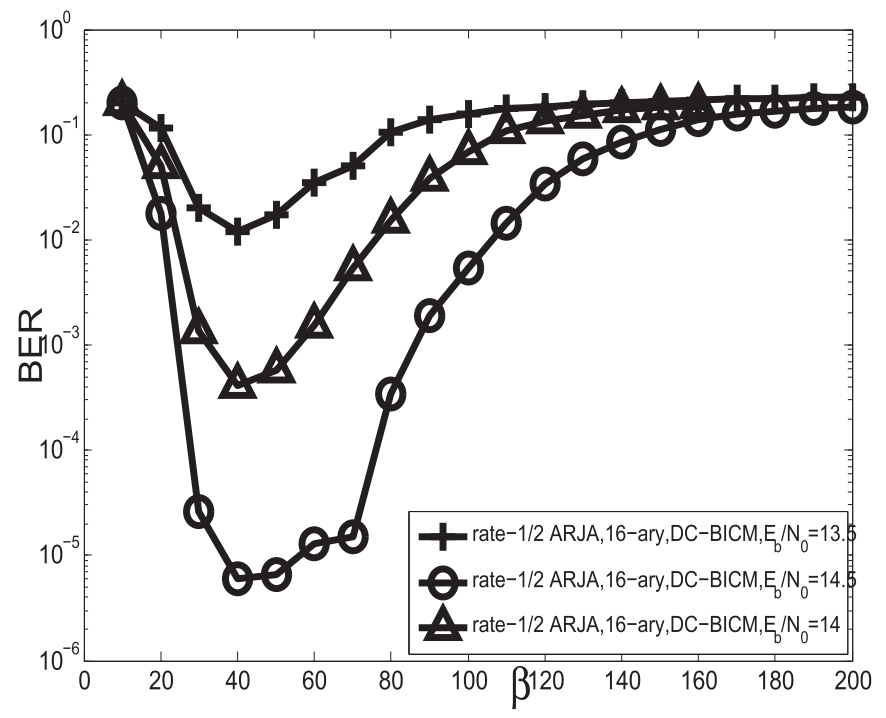

Fig. 9. The impact of $\beta$ on the performance of the rate-1/2 ARJA 16-ary DC-BICM system with different values of $E_{b} / N_{0}$.

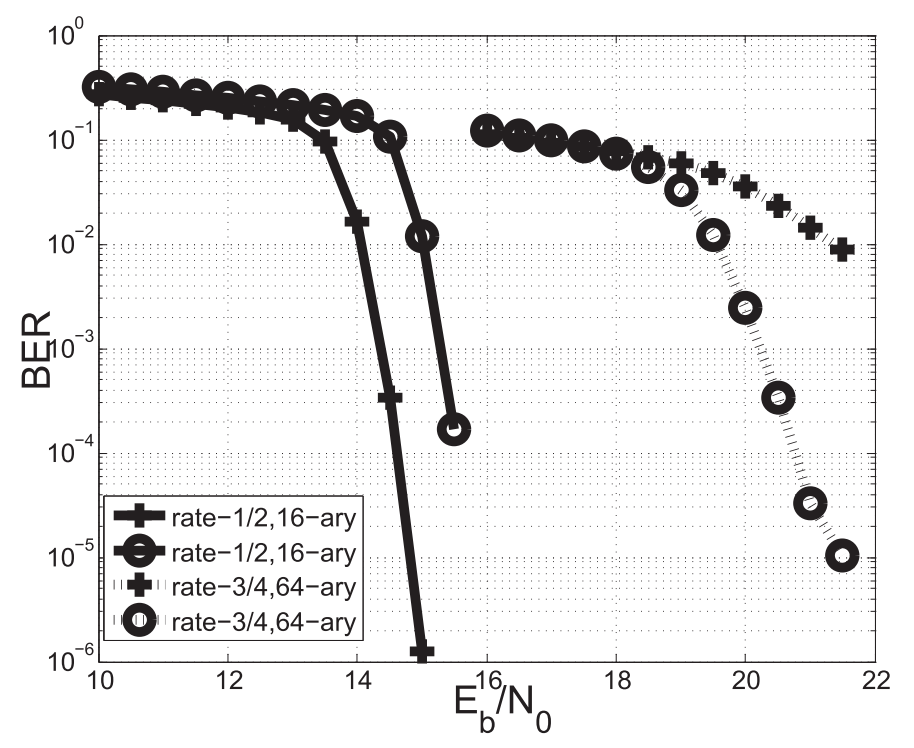

Fig. 10. The performance of the ARJA coded DC-BICM system with different $M$, where the curves labeled by the circle symbol denote $\beta$ is 128 and the curves labeled by plus symbol denote $\beta$ is 64 .

indicate that the performance of the system is better with the increasing decoding iterations. However, the performances have not much differences between 50 and 100 iterations. Therefore, a conclusion can be drawn that there exists best iterations.

Thirdly, the impact of the spreading factor $\beta$ on the performance of the proposed system can be seen from Fig.9. When $\beta$ is smaller than a certain threshold value, the performance gets better and better as its increases, This is because intersymbol interference can not be ignored when the spreading factor is not very large. It is just opposite when $\beta$ is larger than this threshold value. This is in accordance with the performance analysis of s-c-b M-ary DCSK in [9]. Therefore, in the proposed system, there exists its own best spreading factor with different values of $E_{b} / N_{0}$. Moreover, It can be seen

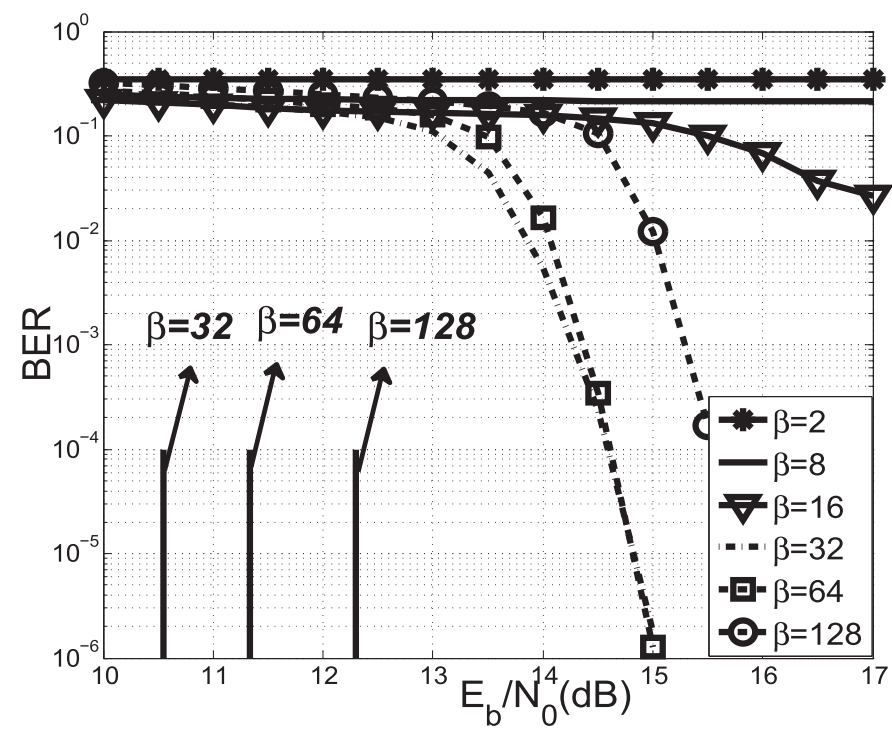

Fig. 11. Comparison between simulation results and threshold values for the DC-BICM system with different spreading factors.

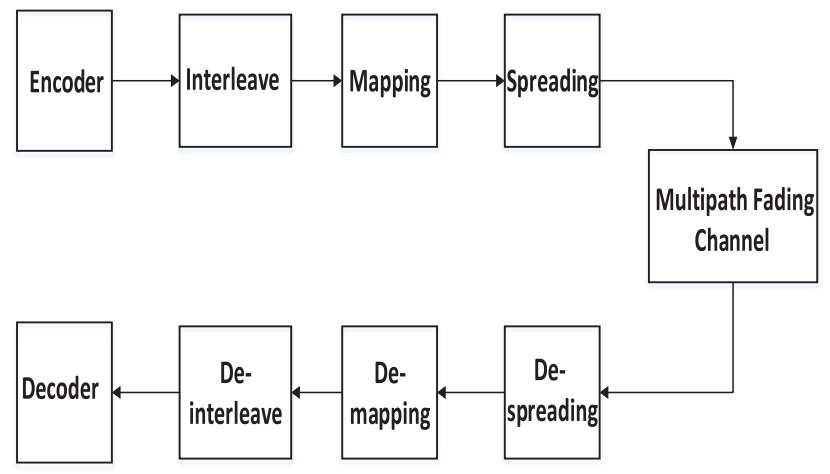

Fig. 12. Block diagram of the BICM DS/SS system.

TABLE I

Thredsholds Calculated By the P-EXIT, the MaXimum NUmber of ITERATION IS SET to 100

\begin{tabular}{lccc}
\hline$\beta$ & 32 & 64 & 128 \\
\hline$\left(E_{b} / N_{0}\right)^{*}$ & 10.543 & 11.334 & 12.302 \\
\hline
\end{tabular}

from Fig.10 that when $M$ is larger, the system performance becomes worse.

Fourthly, the BER versus $E_{b} / N_{0}$ performances for the rate-1/2 ARJA 16-ary DC-BICM system with different spreading factors are depicted in Fig.11, where the maximum number of decoding iterations is set to 50. And, accordingly, the calculated decoding thresholds with different spreading factors according to the P-EXIT analysis are shown in Table.1, and are also plotted in Fig.11. It can be seen that there is a big gap between the simulation results and the analytical values, from which it is found that the system still has a large room for optimization.

Finally, a comparison between DC-BICM and traditional BICM DS/SS systems is given. The block diagram of the BICM DS/SS system is shown in Fig.12, where the 


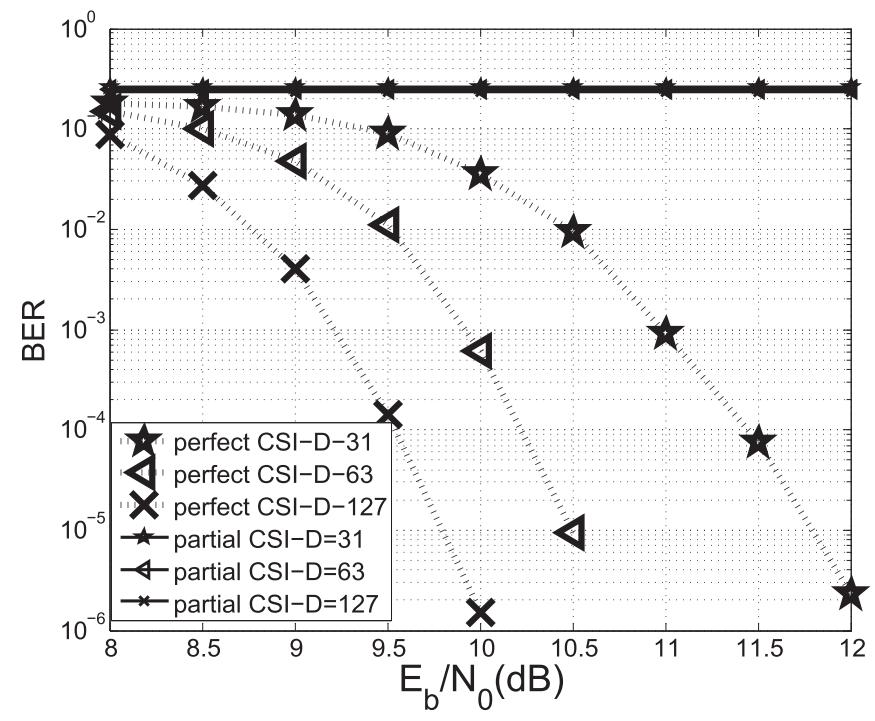

(a) BICM DS/SS system.

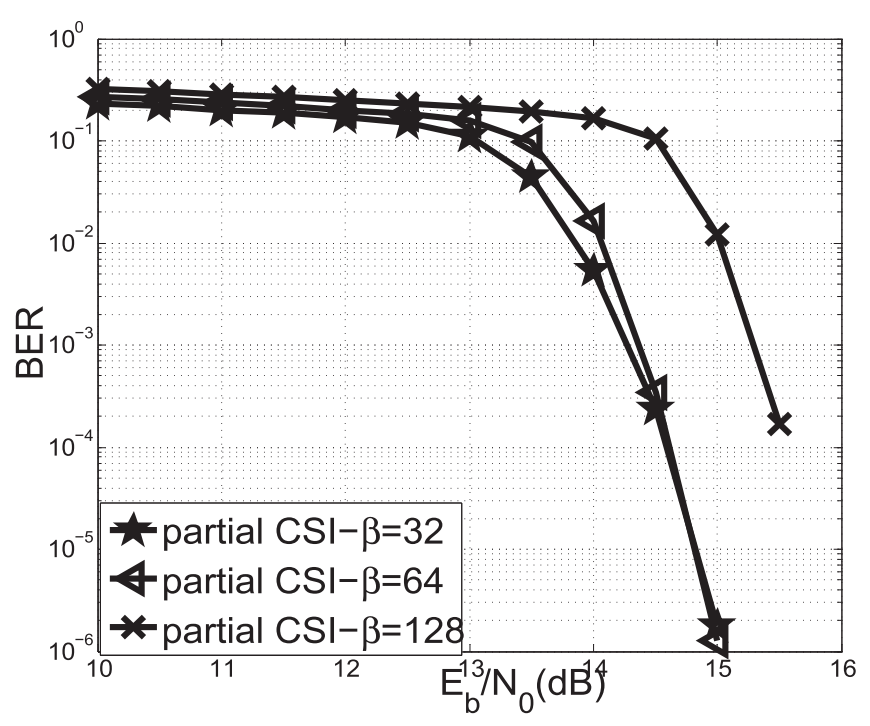

(b) DC-BICM system.

Fig. 13. Performance comparison between the BICM DS/SS system and the DC-BICM system with 50 decoding iterations and 2500 code length.

M sequence is selected as the spreading sequence. The BICM DS/SS system chooses the same code and mapping scheme as DC-BICM, the modulation is the 16-ary QAM.

As can be seen from Fig.13, the BICM DS/SS system has better performance with a perfect CSI, but cannot work with partial CSI compared to the proposed system. From system implementation, the BICM DS/SS system has high demands on synchronization, it is necessary to obtain every channel coefficient. However, the DC-BICM system not only can achieve the channel power value, but also has a simple structure, and its performance is not bad with the optimal spreading factor. Therefore, for those scenarios without very accurate CSI and complex equipment, the proposed system is a better choice.

\section{CONCLUSION}

In this paper, a simple generalized DC-BICM system is designed. The comparisons between the DC-BICM and the squared-constellation-based $M$-ary DCSK systems with same spectral efficiency verify that the proposed system has high coding gain without requiring additional bandwidths and has excellent performance of anti-multipath fading. At the same time, BER simulations show that there exist the best spreading factor and best iterative decoding value for performance of the proposed system, and the performance becomes better with the increasing code length, but being worse with increasing $\mathrm{M}$. Furthermore, simulation results comparing with the threshold values calculated by P-EXIT analysis demonstrate that the system still has a large room for optimization. Finally, a comparison between the DC-BICM and the BICM DS/SS systems reveals that the proposed system has lower complexity and better performance with partial CSI.

Hence, it is concluded that the proposed system offers a good anti-multipath fading performance, high spectrum efficiency, simplicity and robutness thanks to the inheriting advantages of chaotic modulation and BICM technology, and is suitable for band-limited transmission scenarios with ambition performance of anti multi-path attenuation, low complexity and without requiring accurate CSI.

Future work will focus on optimization design of this proposed system and finding more practical applications.

\section{REFERENCES}

[1] G. Kolumbán, B. Vizvári, W. Schwarz, and A. Abel, "Differential chaos shift keying: A robust coding for chaos communication," in Proc. Int. Workshop Nonlinear Dyn. Electron. Syst., Seville, Spain, Jun. 1996, pp. 87-92.

[2] G. Kolumbán, G. Kis, M. P. Kennnedy, and Z. áko, "FM-DCSK: A new and robust solution to chaos communications," in Proc. Int. Symp. Nonlinear Theory, Geneva, Switzerland, 1997, pp. 117-120.

[3] Y. Xia, C. K. Tse, and F. C. M. Lau, "Performance of differential chaosshift-keying digital communication systems over a multipath fading channel with delay spread," IEEE Trans. Circuits Syst. II, Exp. Briefs, vol. 51, no. 12, pp. 680-684, Dec. 2004.

[4] Y. Fang, J. Xu, L. Wang, and G. Chen, "Performance of MIMO relay DCSK-CD systems over Nakagami fading channels," IEEE Trans. Circuits Syst. I, Reg. Papers, vol. 60, no. 3, pp. 757-767, Mar. 2013.

[5] G. Kolumbán, M. Kennedy, and G. Kis, "Multilevel differential chaos shift keying," in Proc. NDES, Moscow, Russia, Jun. 1997, pp. 191-196.

[6] G. Kolumbán and G. Kis, "Reception of $M$-ary FM-DCSK signals by energy detector," in Proc. NDES, Scuol, Switzerland, May 2003, pp. 133-136.

[7] T. Huang, L. Wang, W. Xu, and F. C. M. Lau, "Multilevel code-shifted differential-chaos-shift-keying system," IET Commun., vol. 10, no. 10, pp. 1189-1195, Feb. 2016.

[8] H. Yang, W. K. S. Tang, G. Chen, and G.-P. Jiang, "System design and performance analysis of orthogonal multi-level differential chaos shift keying modulation scheme," IEEE Trans. Circuits Syst. I, Reg. Papers, vol. 63, no. 1, pp. 146-156, Jan. 2016.

[9] G. Cai, Y. Fang, G. Han, F. C. M. Lau, and L. Wang, "A squareconstellation-based $M$-ary DCSK communication system," IEEE Access, vol. 4, pp. 6295-6303, Sep. 2016.

[10] Z. Galias and G. M. Maggio, "Quadrature chaos-shift keying: Theory and performance analysis," IEEE Trans. Circuits Syst. I, Fundam. Theory Appl., vol. 48, no. 12, pp. 1510-1519, Dec. 2001.

[11] S. Wang and X. Wang, " $M$-DCSK-based chaotic communications in MIMO multipath channels with no channel state information," IEEE Trans. Circuits Syst. II, Exp. Briefs, vol. 57, no. 12, pp. 1001-1005, Dec. 2010. 
[12] L. Wang, G. Cai, and G. R. Chen, "Design and performance analysis of a new multiresolution $M$-ary differential chaos shift keying communication system," IEEE Trans. Wireless Commun., vol. 14, no. 9, pp. 5197-5208, Sep. 2015.

[13] G. Kaddoum and F. Gagnon, "Error correction codes for secure chaosbased communication system," in Proc. 25th Biennial Symp Commun., Kingston, ON, Canada, May 2010, pp. 193-196.

[14] L. Wang and G. Chen, "Using LDPC codes to enhance the performance of FM-DCSK," in Proc. 47th Midwest Symp. Circuits Syst., vol. 1. Hong Kong, Jul. 2004, pp. 401-404.

[15] Y. Lyu, L. Wang, G. Cai, and G. Chen, "Iterative receiver for $M$-ary DCSK systems," IEEE Trans. Commun, vol. 63, no. 11, pp. 3929-3936, Nov. 2015.

[16] Y. Lyu, G. Cai, and L. Wang, "Iterative demodulation and decoding of LDPC-coded $M$-ary DCSK modulation over AWGN channel," in Proc. Int. ISMICT, Florence, Italy, Apr. 2014, pp. 1-5.

[17] C. Zhang, L. Wang, and G. Chen, "Promising performance of PA-coded SIMO FM-DCSK communication systems," Circuits, Syst. Signal Process., vol. 27, no. 6, pp. 915-926, Nov. 2008.

[18] A. Abbasfar, D. Divsalar, and K. Yao, "Accumulate-repeat-accumulate codes," IEEE Trans. Commun., vol. 55, no. 4, pp. 692-702, Apr. 2007.

[19] E. Zehavi, "8-PSK trellis codes for a Rayleigh channel," IEEE Trans. Commun., vol. 40, no. 5, pp. 873-884, May 1992.

[20] G. Caire, G. Taricco, and E. Biglieri, "Bit-interleaved coded modulation," IEEE Trans. Inf. Theory, vol. 44, no. 3, pp. 927-946, May 1998.

[21] J. Li, Y. Yang, H. Yu, and Y. Guan, "LDPC coded modulation for Rayleigh fading channels," in Proc. Int. WCSP, Shanghai, China, Oct. 2012, pp. 1-5.

[22] D. Divsalar and C. Jones, "Protograph based low error floor LDPC coded modulation," in Proc. IEEE MILCOM, Pasadena, CA, USA, Oct. 2005, pp. 378-385.

[23] Y. Jin, M. Jiang, and C. Zhao, "Optimized variable degree matched mapping for protograph LDPC coded modulation with 16QAM," in Proc. IEEE ISTC, Brest, France, May 2014, pp. 853-856.

[24] C. Tang, H. Shen, M. Jiang, and C. Zhao, "Optimization of generalized VDMM for protograph-based LDPC coded BICM," IEEE Commun. Lett., vol. 18, no. 5, pp. 853-856, May 2014.

[25] C.-H. Yihand and E. Geraniotis, "Iterative multiuser detection for bitinterleaved coded modulation based CDMA signals in fading channels," in Proc. IEEE ISSSTA, Prague, Czech Republic, Sep. 2002, pp. 485-489.

[26] U. Wachsmann, R. F. H. Fischer, and J. B. Huber, "Multilevel codes: Theoretical concepts and practical design rules," IEEE Trans. Inf. Theroy, vol. 45, no. 5, pp. 1361-1391, Jul. 1999.

[27] S. ten Brink, G. Kramer, and A. Ashikhmin, "Design of lowdensity parity-check codes for modulation and detection," IEEE Trans. Commun., vol. 52, no. 4, pp. 670-678, Apr. 2004.

[28] Y. Fang, P. Chen, L. Wang, and F. C. M. Lau, "Design of protograph LDPC codes for partial response channels," IEEE Trans. Commun., vol. 60 , no. 10 , pp. 2809-2819, Oct. 2012.

[29] D. Divsalar, S. Dolinar, C. R. Jones, and K. Andrews, "Capacityapproaching protograph codes," IEEE J. Sel. Areas Commun., vol. 27, no. 6, pp. 876-888, Aug. 2009.

[30] R. Lucas, M. P. C. Fossorier, Y. Kou, and S. Lin, "Iterative decoding of one-step majority logic deductible codes based on belief propagation," IEEE Trans. Commun., vol. 48, no. 6, pp. 931-937, Jun. 2000.

[31] D. F. Tseng and K. C. Lu, "Iterative low-complexity multi-user detection for asynchronous multi-sequence signalling based bit-interleaved coded modulation code division multiple access systems," IET Commun., vol. 1, no. 4, pp. 628-637, Aug. 2007. 